\title{
ON THE GREATEST PRIME FACTORS OF DECOMPOSABLE FORMS AT INTEGER POINTS
}

\section{K. GYŌRY}

\section{Introduction}

Let $f \in \boldsymbol{Z}[x, y]$ be a binary form and assume that among the linear factors in the factorization of $f$ at least three are distinct. Mahler [12] proved that $P(f(x, y)) \rightarrow \infty$ if $X=\max (|x|,|y|) \rightarrow \infty$ with $x, y \in \boldsymbol{Z},(x, y)=1$, where $P(n)$ denotes the greatest prime factor of $n$. Mahler's work was generalized by Parry [14]. For irreducible forms $f$ Coates [4] improved Mahler's result by showing that if $\alpha=1 / 4$, then for any coprime integers $x, y$

$$
P(f(x, y))>c_{1}(\log \log X)^{\alpha}, \quad X \geqq X_{1},
$$

where $c_{1}>0$ and $X_{1}>0$ depend only on $f$ and can be given explicitly. Sprindžuk [21], [22] established (1) with $\alpha=1$ for all such forms of degree at least 5 and for so-called non-exceptional forms of degree 4. Kotov [11] generalized Sprindžuk's result to binary forms with algebraic integer coefficients. Shorey, van der Poorten, Tijdeman and Schinzel [20] proved that if $f \in \boldsymbol{Z}[x, y]$ has at least three distinct linear factors in its factorization and $\alpha=1$, then (1) holds for any $x, y \in \boldsymbol{Z}$ with $(x, y)=d$, where $d$ is a fixed positive integer .

Schlickewei [17], [18] proved that for a large class of norm forms $F \in \boldsymbol{Z}\left[x_{1}, \ldots, x_{m}\right]$ in $m \geqq 2$ variables and for $\mathbf{x}=\left(x_{1}, \ldots, x_{m}\right) \in \boldsymbol{Z}^{m}$ with relatively prime components, $P(F(\mathbf{x})) \rightarrow \infty$ as $|\mathbf{x}|=\max \left(\left|x_{1}\right|, \ldots,\left|x_{m}\right|\right) \rightarrow \infty$. For index forms $F \in \boldsymbol{Z}\left[x_{1}, \ldots, x_{m}\right]$ Trelina [24] showed that

$$
P(F(\mathbf{x}))>c_{2}(\log \log |\mathbf{x}| \log \log \log |\mathbf{x}|)^{1 / 2}, \quad|\mathbf{x}| \geqq X_{2} .
$$

Independently, for discriminant forms and index forms $F \in \boldsymbol{Z}\left[x_{1}, \ldots, x_{m}\right]$

$$
P(F(\mathbf{x}))>c_{3} \log \log |\mathbf{x}|, \quad|\mathbf{x}| \geqq X_{3},
$$

have been established by Papp and the author [8]. Here $\mathbf{x} \in \boldsymbol{Z}^{m}$ with $\left(x_{1}, \ldots, x_{m}\right)=1$ and $c_{2}, c_{3}, X_{2}, X_{3}$ are effectively computable positive numbers depending only on $F$. Recently the author [10] proved (2) for a wide class of irreducible norm forms $F(\mathbf{x})$ in $m \geqq 2$ variables (including all binary forms). In [8] and [10] our estimates are established for forms $F(\mathbf{x}) \in \boldsymbol{Z}_{L}\left[x_{1}, \ldots, x_{m}\right]$ at integer points $\mathbf{x} \in \boldsymbol{Z}_{L}^{m}$, where $\boldsymbol{Z}_{L}$ denotes the ring of integers of an arbitrary but fixed algebraic number field $L$. 
In this paper we give a common generalization of our results mentioned above and compute an explicit value of the constant corresponding to $c_{3}$. Our main result implies the above-quoted theorems of Sprindžuk [21], [22], Kotov [11], Shorey, van der Poorten, Tijdeman and Schinzel [20], Trelina [24], Győry and Papp [8] and Győry [10].

\section{Results}

Before we state our theorem, we establish our notation and introduce some definitions.

A system $\mathscr{L}$ of $n \geqq 2$ linear forms $L_{1}(\mathbf{x}), \ldots, L_{n}(\mathbf{x})$ in $\mathbf{x}=\left(x_{1}, \ldots, x_{m}\right)$ with algebraic coefficients will be called triangularly connected or, more briefly, $\Delta$-connected (cf. [7]) if for any distinct $i, j$ with $1 \leqq i, j \leqq n$ there is a sequence $L_{i}=L_{i_{1}}, \ldots, L_{i_{v}}=L_{j}$ in $\mathscr{L}$ such that for each $u$ with $1 \leqq u \leqq v-1, L_{i_{u}}, L_{i_{u+1}}$ have a linear combination with non-zero algebraic coefficients which belongs to $\mathscr{L}$. If in particular $m=2$, then every system $\mathscr{L}$ which contains at least three pairwise non-proportional linear forms is $\Delta$-connected.

Throughout the paper, $L$ will denote a fixed algebraic number field of degree $l \geqq 1$ with ring of integers $Z_{L}$, and $U_{L}$ will be the group of units in $L$. We denote by $\omega(\alpha)$ the number of distinct prime ideal divisors $\mathfrak{p}$ of a non-zero integer $\alpha$ in $L$ and by $\mathscr{P}(\alpha)$ the greatest of the norms $N(\mathfrak{p})$ of these prime ideals. For $\alpha \in U_{L}$ we take $\mathscr{P}(\alpha)=1$ and $\omega(\alpha)=0$.

If $F\left(x_{1}, \ldots, x_{m}\right) \in \boldsymbol{Z}_{L}\left[x_{1}, \ldots, x_{m}\right]$ is a form in $m \geqq 2$ variables, then $F\left(x_{1}, \ldots, x_{m}\right)$ and $F\left(\varepsilon x_{1}, \ldots, \varepsilon x_{m}\right)$ have the same prime ideal decomposition for any $\mathbf{x}=$ $\left(x_{1}, \ldots, x_{m}\right) \in \boldsymbol{Z}_{L}^{m}$ and $\varepsilon \in U_{L}$. It will be useful to introduce the notation $\overline{|\mathbf{x}|}$ defined by $\left.{ }^{1}\right)$

$$
\overline{|\mathbf{x}|}=\min _{\varepsilon \in U_{L}} \max \left(\overline{\mid \varepsilon x_{1}}\left|, \ldots, \overline{\mid \varepsilon x_{m}}\right|\right), \quad m \geqq 2,
$$

where $\mathbf{x}=\left(x_{1}, \ldots, x_{m}\right) \in \boldsymbol{Z}_{L}^{m} . \quad s_{0}|\overline{\mathbf{x}}|$ can be effectively determined and clearly

$$
N^{1 / l} \leqq \overline{|\mathbf{x}|} \leqq \max \left(\overline{\left|x_{1}\right|}, \ldots, \overline{\left|x_{m}\right|}\right)
$$

for any $\mathbf{x} \in \boldsymbol{Z}_{L}^{m}$, where $N=\max _{1 \leqq i \leqq m}\left(\left|N_{L / Q}\left(x_{i}\right)\right|\right)$. Further, it is clear that in the special case $L=\boldsymbol{Q} \overline{|\mathbf{x}|}$ coincides with $|\mathbf{x}|$.

Our main result is the following

Theorem. Let $F(\mathbf{x})=F\left(x_{1}, \ldots, x_{m}\right) \in \boldsymbol{Z}_{L}\left[x_{1}, \ldots, x_{m}\right]$ be a decomposable form of degree $n \geqq 3$ in $m \geqq 2$ variables with splitting field $G$ over $L$, and let $[G: Q]=g$, $[G: L]=f$. Suppose that the linear factors $L_{1}(\mathbf{x}), \ldots, L_{n}(\mathbf{x})$ in the factorization of

1) $\overline{|\gamma|}$ denotes the maximum absolute value of the conjugates of an algebraic number $\gamma$. 
$F$ form a $\Delta$-connected system and that there is no $0 \neq \mathbf{x} \in L^{m}$ for which $L_{j}(\mathbf{x})=0$, $j=1, \ldots, n$. Let $d$ be a positive integer. Then there exists an effectively computable number $X_{4}$ depending only on $F, d$ and $L$, such that

$$
(13 f+1) s \log (s+1)+(g+1) \log \mathscr{P}>\log \log |\overline{\mathbf{x}}|
$$

and

$$
\mathscr{P}>((13 f+1) l)^{-\alpha}(\log \log \overline{\mid \mathbf{x}} \mid)^{\alpha}
$$

for any $\quad \mathbf{x} \in \boldsymbol{Z}_{L}^{m}$ with $N\left(\left(x_{1}, \ldots, x_{m}\right)\right) \leqq d$ and $\overline{|\mathbf{x}|} \geqq X_{4}$, where $\mathscr{P}=\mathscr{P}\left(F^{\prime}(\mathbf{x})\right)$, $s=\omega(F(\mathbf{x})), \mathscr{P}=P^{\alpha}$ and $P$ is the maximal rational prime for which $(F(\mathbf{x}), P) \neq 1$.

It is easily seen that under the conditions and notations of the theorem we have $1 \leqq \alpha \leqq l$,

and

$$
(13 f+1) s \log (s+1)+(g+1) \log \mathscr{P}>\log \log N
$$

$$
\mathscr{P}>((13 f+1) l)^{-\alpha}(\log \log N)^{\alpha}
$$

for any $\mathbf{x} \in Z_{L}^{m}$ with $N\left(\left(x_{1}, \ldots, x_{m}\right)\right) \leqq d$ and $N=\max _{1 \leqq i \leqq m}\left(\left|N_{L / Q}\left(x_{i}\right)\right|\right) \geqq N_{1}$. For small values of $s$ the estimates (4) and (4') are obviously much better than (5) and (5').

Our theorem has several consequences. We first mention an application to diophantine equations. Let $F(\mathbf{x})$ and $d$ be as in the theorem and let $\beta, \pi_{1}, \ldots, \pi_{t}$ be fixed non-zero algebraic integers in $L$. Consider the equation

$$
F(\mathbf{x})=\beta \pi_{1}^{z_{1}} \ldots \pi_{t}^{z_{t}}
$$

in $\mathbf{x} \in \boldsymbol{Z}_{L}^{m}, z_{1}, \ldots, z_{t} \in \boldsymbol{Z}$ with $N\left(\left(x_{1}, \ldots, x_{m}\right)\right) \leqq d$ and $z_{1}, \ldots, z_{t} \geqq 0$. Then (4) gives

$$
\max \left(\overline{|\mathbf{x}|}, e^{\max _{k}\left(z_{k}\right)}\right)<C
$$

for all solutions $\mathbf{x}, z_{1}, \ldots, z_{t}$ of (6), where $C$ is an effectively computable number ${ }^{2}$ ) depending only on $F, d, \mathscr{P}\left(\beta \pi_{1} \ldots \pi_{t}\right), \omega\left(\beta \pi_{1} \ldots \pi_{t}\right)$ and $L$. This result can be regarded as a $\mathfrak{p}$-adic analogue of our Theorem 1 in [7]. (In [7] it is not assumed $F \in \boldsymbol{Z}_{L}[\mathbf{x}]$; however, in the applications of Theorem 1 of [7] $F \in \boldsymbol{Z}_{L}[\mathbf{x}]$ is always supposed. Thus this is not an essential restriction.)

The following corollary enables us to obtain some information about the arithmetical structure of those algebraic integers of $L$ which can be represented by a decomposable form of the above type.

Corollary 1. Suppose $F\left(x_{1}, \ldots, x_{m}\right)$ and $d$ are as in the Theorem. Let $F$ be any algebraic integer in $L$ represented by $F\left(x_{1}, \ldots, x_{m}\right)$, where $x_{1}, \ldots, x_{m} \in Z_{L}$ with $N\left(\left(x_{1}, \ldots, x_{m}\right)\right) \leqq d$. Then

$$
(13 f+1) \omega(F) \log (\omega(F)+1)+(g+1) \log \mathscr{P}(F)>\log \log N
$$

$\left.{ }^{2}\right)$ We could easily obtain an explicit expression for $C$ by computing each constant in the proof of our theorem. Added in proof: In my paper, ,Explicit upper bounds for the solutions of some diophantine equations" (to appear) I explicitly evaluated $C$ in terms of each constant, (generalizing many earlier effective results on norm form, discriminant form and index form equations). 
and

$$
\mathscr{P}(F)>((13 f+1) l)^{-1} \log \log N
$$

if $N=\left|N_{L / Q}(F)\right| \geqq N_{2}$, where $N_{2}$ is an effectively computable positive number depending only on $d, L$ and the form $F\left(x_{1}, \ldots, x_{m}\right)$.

Our Corollary 1 generalizes and improves Sprindžuk's theorems [22], [23] concerning rational integers represented by a binary form $f \in Z[x, y]$.

Corollary 2. Let $F(\mathbf{x}) \in Z_{L}\left[x_{1}, \ldots, x_{m}\right]$ be a decomposable form with the properties specified in the Theorem. Let $d$ and $A$ be positive numbers with $d \geqq 1$ and $A<1 /(g+1)$. Then there exists an effectively computable number $X_{5}$ depending only on $F, d, L$ and $A$ such that if

$$
\mathscr{P}(F(\mathbf{x})) \leqq(\log |\overline{\mathbf{x}}|)^{A}, \quad \mathbf{x} \in Z_{L}^{m}, \quad \overline{\mid \mathbf{x}} \mid \geqq X_{5}
$$

and $N\left(\left(x_{1}, \ldots, x_{m}\right)\right) \leqq d$, then

where $c_{4}=(1-A(g+1)) /(13 f+1)$.

$$
\omega(F(\mathbf{x}))>c_{4} \frac{\log \log \overline{|\mathbf{x}|}}{\log \log \log \overline{|\mathbf{x}|}},
$$

Let $f \in Z_{L}[x]$ be a polynomial with at least three distinct roots. Since $|\bar{x}|^{1 / l} \leqq$ $\max (\overline{|\varepsilon x|}, \overline{|\varepsilon|})$ for any $x \in Z_{L}$ and $\varepsilon \in U_{L}$, our estimates (4), (5), (7), (8) and (9) remain obviously valid for $\mathscr{P}(f(x))$ and $\omega(f(x))$ with $\overline{|x|}$ instead of $\overline{|\mathbf{x}|}$, where $x \in \boldsymbol{Z}_{L}$ and $\overline{|x|}>X_{6}$. We remark that for polynomials $f(x)$ with rational integer coefficients Shorey and Tijdeman [19] obtained a much better result than our Corollary 2; they proved $\omega(f(x)) \gg(\log \log |x|) /(\log \log \log |x|)$ under the condition $P(f(x)) \leqq \exp \left((\log \log |x|)^{A}\right)$, where $A$ is any positive number. As an immediate consequence of this result they derived a good lower bound for $\max _{1 \leqq i \leqq y} P(f(x+i))$.

As a consequence of our theorem we obtain the following generalization and improvement, respectively, of the theorems of Coates [4], Sprindžuk [21], [22], Kotov [11] and Shorey, van der Poorten, Tijdeman and Schinzel [20] on the maximal prime factors of binary forms.

Corollary 3. Let $f(x, y) \in \boldsymbol{Z}_{L}[x, y]$ be a binary form with splitting field $G$ over $L$ and suppose that among the linear factors in the factorization of $f$ at least three are distinct $\left.^{3}\right)$. Let $[G: Q]=g,[G: L]=f$ and $d \geqq 1$. Then there exists an effectively computable positive number $X_{7}$ depending only on $d, L$ and the form $f(x, y)$ such that for all pairs $x, y \in \boldsymbol{Z}_{L}$ with $N((x, y)) \leqq d$ and $\overline{|\mathbf{x}|}=\min _{\varepsilon \in U_{L}} \max (\overline{|\varepsilon x|}, \overline{\varepsilon y \mid})>X_{7}$, (4) and (5) hold, where $\mathscr{P}=\mathscr{P}(f(x, y)), s=\omega(f(x, y)), \mathscr{P}=P^{\alpha}$ and $P$ is the maximal rational prime with $(f(x, y), P) \neq 1$.

${ }^{3}$ ) In other words $f$ has at least three pairwise nonproportional linear factors in its factorization. 
It follows from $\left(5^{\prime}\right)$ that

$$
\mathscr{P}(f(x, y))>c_{5}(\log \log N)^{\alpha}
$$

for all $x, y \in \boldsymbol{Z}_{L}$ with $(x, y)=1$ and $N=\max \left(\left|N_{L / Q}(x)\right|,\left|N_{L / Q}(y)\right|\right) \geqq N_{3}$, where $c_{5}=((13 f+1) l)^{-\alpha}$. For irreducible forms $f \in \boldsymbol{Z}_{L}[x, y]$ of degree $\geqq 5$ (10) was earlier proved by Kotov [11].

An important special case of Corollary 3 is when $f(x, y)=\left(x-\alpha_{1} y\right) \ldots\left(x-\alpha_{n} y\right)$, where $\alpha_{1}, \ldots, \alpha_{n} \in Z_{L}$ and at least three of them are distinct. This special case of Corollary 3 can be used to obtain an effective result on the diophantine equation $a z^{q}=f(x, y)$ (cf. [20], pp. 63-65).

Corollary 4. Let $K$ be an extension of degree $n \geqq 3$ of $L$ and let $F(\mathbf{x})=$ $a_{0} N_{K / L}\left(x_{1}+\alpha_{2} x_{2}+\ldots+\alpha_{m} x_{m}\right) \in Z_{L}\left[x_{1}, \ldots, x_{m}\right]$ be a norm form in $m \geqq 2$ variables such that $\left[L\left(\alpha_{i}\right): L\right]=n_{i} \geqq 3, i=2, \ldots, m$, and $n_{2} \ldots n_{m}=n$. Then with the notations of the Theorem we have (4) and (5).

Corollary 4 implies Corollary 2 of [10] and Theorem 3 of Kotov [11].

Corollary 5. Let $K$ be as in Corollary 4. Let $\alpha_{1}, \ldots, \alpha_{m}$ be $m \geqq 2$ algebraic integers in $K$ with $K=L\left(\alpha_{1}, \ldots, \alpha_{m}\right)$ and suppose that $1, \alpha_{1}, \ldots, \alpha_{m}$ are linearly independent over L. Let $F(\mathbf{x})$ denote the discriminant form $\operatorname{Discr}_{K / L}\left(\alpha_{1} x_{1}+\ldots+\alpha_{m} x_{m}\right)$. Under the notations of the Theorem, for $F(\mathbf{x})(4)$ and (5) hold.

Corollary 5 improves Corollary 1 of our paper [8].

Let again $K$ be an extension of degree $n \geqq 3$ of $L$ and let $G$ be the smallest normal extension of $L$ containing $K$. Write $[G: Q]=g$ and $[G: L]=f$. Consider an order $O$ of the field extension $K / L$ (i.e. a subring of $\boldsymbol{Z}_{K}$ containing $\boldsymbol{Z}_{L}$ that has the full dimension $n$ as a $Z_{L}$-module) and suppose that $O$ has a relative integral basis $1, \alpha_{1}, \ldots, \alpha_{n-1}$ over $L$. (Such an integral basis exists for a number of orders of $K / L$; see e.g. [2], [13] and [8].) Then we have (cf. [8])

$\operatorname{Discr}_{K / L}\left(\alpha_{1} x_{1}+\ldots+\alpha_{n-1} x_{n-1}\right)=\left[\operatorname{Ind}_{K / L}\left(\alpha_{1} x_{1}+\ldots+\alpha_{n-1} x_{n-1}\right)\right]^{2} D_{K / L}\left(1, \alpha_{1}, \ldots, \alpha_{n-1}\right)$, where $I(x)=\operatorname{Ind}_{K / L}\left(\alpha_{1} x_{1}+\ldots+\alpha_{n-1} x_{n-1}\right) \in Z_{L}\left[x_{1}, \ldots, x_{n-1}\right]$ is a decomposable form of degree $n(n-1) / 2$. It is called the index form of the basis $1, \alpha_{1}, \ldots, \alpha_{n-1}$ of $O$ over $L$.

In the special case $L=Q$ Trelina [24] obtained lower bounds for $P(I(\mathbf{x}))$. Corollary 1 and Theorem 3 in our paper [8], established independently of Trelina, give lower bounds for $\mathscr{P}(I(\mathbf{x}))$ in the above general case. As a consequence of Corollary 5 we obtain the following generalization and improvement of the estimates of Trelina [24] and Győry and Papp [8]. 
Corollary 6. Let $L, K, d$ and $I(\mathbf{x})$ be defined as above. Then there exists an effectively computable positive number $X_{8}$ depending only on $I(\mathbf{x}), d, L$ and $D_{K / L}\left(1, \alpha_{1}, \ldots, \alpha_{n-1}\right)$ such that (4) and (5) hold for any $\mathbf{x} \in Z_{L}^{n-1}$ with $N\left(\left(x_{1}, \ldots, x_{n-1}\right)\right) \leqq d$ and $\overline{|\mathbf{x}|} \geqq X_{8}$, where $\mathscr{P}=\mathscr{P}(I(\mathbf{x})), s=\omega\left(I(\mathbf{x}) D_{K / L}\left(1, \alpha_{2}, \ldots, \alpha_{n-1}\right)\right), \mathscr{P}=P^{\alpha}$ and $P$ is the maximal rational prime with $(I(\mathbf{x}), P) \neq 1$.

The proof of our theorem depends on two deep theorems, due to van der Poorten and Loxton [16] and van der Poorten [15], which are essentially sharp inequalities on linear forms in the complex and in the $\mathfrak{p}$-adic case.

\section{Proof of the Theorem}

We first show that we can make certain assumptions without loss of generality. By using a well-known argument we can easily see that there exist algebraic integers $a_{2}, \ldots, a_{m}$ in $L$ such that $F\left(1, a_{2}, \ldots, a_{m}\right) \neq 0$ (see e.g. [3], p. 77). It suffices to prove the theorem for $F\left(x_{1}, a_{2} x_{1}+x_{2}, \ldots, a_{m} x_{1}+x_{m}\right)$, where the coefficient of $x_{1}^{n}$ is nonzero. Hence we may suppose that

$$
F(\mathbf{x})=a_{0} L_{1}(\mathbf{x}) \ldots L_{n}(\mathbf{x})
$$

with $0 \neq a_{0} \in Z_{L}$ and

$$
L_{j}(\mathbf{x})=x_{1}+\alpha_{2 j} x_{2}+\ldots+\alpha_{m j} x_{m}, j=1, \ldots, n,
$$

where $\alpha_{i j} \in G, 2 \leqq i \leqq m, 1 \leqq j \leqq n$. Writing $\alpha_{i j}^{\prime}=a_{0} \alpha_{i j}$ for $i \geqq 2$ and $\alpha_{i j}^{\prime}=a_{0}$ for $i=1$, we have $\alpha_{i j}^{\prime} \in \boldsymbol{Z}_{G}$ for each $i$ and $j$. We shall prove our theorem for

$$
f(\mathbf{x})=a_{0}^{n-1} F(\mathbf{x})=\prod_{j=1}^{n} L_{j}^{\prime}(\mathbf{x}),
$$

where $L_{j}^{\prime}(\mathbf{x})=\alpha_{1 j}^{\prime} x_{1}+\ldots+\alpha_{m j}^{\prime} x_{m}$. This will imply at once the assertion of the theorem for $F(\mathbf{x})$.

We suppose that there are $r_{1}$ real and $2 r_{2}$ complex conjugate fields to $G$ and that they are chosen in the usual manner: if $\theta$ is in $G$, then $\theta^{(i)}$ is real for $1 \leqq i \leqq r_{1}$ and $\theta^{\left(i+r_{2}\right)}=\overline{\theta^{(i)}}$ for $r_{1}+1 \leqq i \leqq r_{1}+r_{2}$. Put $r=r_{1}+r_{2}-1$. It is well-known that there exist fundamental units $\eta_{1}, \ldots, \eta_{r}$ in $G$ and constants $c_{6}, c_{7}$ such that $|\log | \hat{r}_{h}^{(i)}|| \leqq c_{6}$ for $1 \leqq h \leqq r, 1 \leqq i \leqq g$ and $R_{G}>c_{7}$, where $R_{G}$ denotes the regulator of $G$. Here, and below, $c_{6}, c_{7}, \ldots$ will denote effectively computable positive numbers which depend only on $F(\mathbf{x}), L$ and (some of them) on $d$. d. Put

Let $x_{1}, \ldots, x_{m}$ be any $m$-tuple of algebraic integers in $L$ with $N\left(\left(x_{1}, \ldots, x_{m}\right)\right) \leqq$

$$
\beta_{j}=\alpha_{1 j}^{\prime} x_{1}+\ldots+\alpha_{m j}^{\prime} x_{m}, \quad j=1, \ldots, n,
$$

and

$$
(f(\mathbf{x}))=\left(\beta_{1} \ldots \beta_{n}\right)=\mathfrak{p}_{1}^{v} \ldots \mathfrak{p}_{s}^{v},
$$


where $\mathfrak{p}_{1}, \ldots, \mathfrak{p}_{s}$ are distinct prime ideals in $L$. If $X_{4}$ is sufficiently large and $\overline{|\mathbf{x}|} \geqq X_{4}$, then Theorem 1 of [7] implies $s>0$ and $P>1$. Let $\mathfrak{P}_{1}, \ldots, \mathfrak{P}_{t}$ be all distinct prime ideals in $G$ lying above $\mathfrak{p}_{1}, \ldots, \mathfrak{p}_{s}$. Clearly $t \leqq s f$. Applying now the unique factorization theorem to (13) we get in $\boldsymbol{Z}_{G}$

$$
\left(\beta_{j}\right)=\mathfrak{P}_{1}^{U_{1 j}} \ldots \mathfrak{P}_{t}^{U}{ }_{t j}, \quad j=1, \ldots, n,
$$

where the $U_{k j}$ are non-negative rational integers. Denote by $h_{G}$ the class number of $G$ and write $U_{k j}=h_{G} u_{k j}+r_{k j}$ with $0 \leqq r_{k j}<h_{G}$. We have $\mathfrak{P}_{k}^{h_{G}}=\left(\mu_{k}\right)$ with some $\mu_{k} \in \boldsymbol{Z}_{G}$. Then from (14) we see that

$$
\left(\beta_{j}\right)=\left(\chi_{j}\right)\left(\mu_{1}\right)^{u_{1 j}} \ldots\left(\mu_{t}\right)^{u_{t j}}
$$

where $\left(\chi_{j}\right)=\mathfrak{P}_{1}^{r_{1 j} \ldots \mathfrak{P}_{t}^{r_{t j}} \text { and }}$

$$
\left|N_{G / Q}\left(\mu_{k}\right)\right| \leqq P^{g h_{G}}, \quad\left|N_{G / Q}\left(\chi_{j}\right)\right| \leqq P^{g h_{G} t} .
$$

So, following a well-known argument (see e.g. [1], p. 188), we may choose $\mu_{k}$ and $\chi_{j}$ such that

$$
|\log | \mu_{k}^{(i)}|| \leqq c_{8} \log P, \quad|\log | \chi_{j}^{(i)}|| \leqq c_{8} s \log P, \quad i=1, \ldots, g,
$$

and, by (15), we have

for some unit $\varepsilon_{j}$ of $G$.

$$
\beta_{j}=\varepsilon_{j} \chi_{j} \mu_{1}^{u} \ldots \mu_{t}^{u t}, \quad j=1, \ldots, n,
$$

Put $\mathscr{L}=\left\{L_{1}^{\prime}, \ldots, L_{n}^{\prime}\right\}$. By hypothesis there are two forms in $\mathscr{L}$, say $L_{1}^{\prime}$ and $L_{2}^{\prime}$, such that $\lambda_{1} L_{1}^{\prime}(\mathbf{x})+\lambda_{2} L_{2}^{\prime}(\mathbf{x}) \in \mathscr{L}$ with non-zero algebraic numbers $\lambda_{1}, \lambda_{2}$. Suppose, for convenience, that

$$
\lambda_{1} L_{1}^{\prime}(\mathbf{x})+\lambda_{2} L_{2}^{\prime}(\mathbf{x})+\lambda_{3} L_{3}^{\prime}(\mathbf{x})=0
$$

with $\lambda_{1} \lambda_{2} \lambda_{3} \neq 0$. Further, we may assume that $\lambda_{1}, \lambda_{2}, \lambda_{3} \in \boldsymbol{Z}_{G}$ and $\max \left(\left|\overline{\lambda_{1} \mid}, \overline{\lambda_{2}}\right|, \mid \overline{\lambda_{3} \mid}\right) \leqq$ $c_{9}$. We obtain now

$$
\lambda_{1} \beta_{1}+\lambda_{2} \beta_{2}+\lambda_{3} \beta_{3}=0 .
$$

Put $a_{k}=\min _{q} u_{k q}$ and $u_{k q}^{\prime}=u_{k q}-a_{k}$ for $q=1,2,3$ and $k=1, \ldots, t$. We may suppose without loss of generality that $U=\max _{k, q} u_{k q}^{\prime}=u_{11}^{\prime}$ and $u_{13}^{\prime}=0$. Since $\eta_{1}, \ldots, \eta_{r}$ are fundamental units, we can write

$$
\varepsilon_{1} / \varepsilon_{3}=\varrho_{1} \eta_{1}^{w 11} \ldots \eta_{r}^{w}, \quad \varepsilon_{2} / \varepsilon_{3}=\varrho_{2} \eta_{1}^{w 12} \ldots \eta_{r}^{w},
$$

where $\varrho_{1}, \varrho_{2}$ are roots of unity in $G$ and $w_{11}, \ldots, w_{r 1}, w_{12}, \ldots, w_{r 2}$ are rational integers. With the notation

$$
\beta_{q}=\sigma \delta_{q}, \quad \sigma=\varepsilon_{3} \mu_{1}^{a_{1}} \ldots \mu_{t}^{a_{t}}, \quad \delta_{q}=\chi_{q} \varrho_{q} \eta_{1}^{w_{1 q}} \ldots \eta_{r}^{w_{r q}} \mu_{1}^{u_{1 q}^{\prime}} \ldots \mu_{t}^{u_{t q}^{\prime}}
$$

and $w_{13}=\ldots=w_{\mathrm{r} 3}=0, \varrho_{3}=1$ we get from (17)

$$
\Lambda=-\frac{\lambda_{2} \delta_{2}}{\lambda_{3} \delta_{3}}-1=\frac{\lambda_{1} \delta_{1}}{\lambda_{3} \delta_{3}} \neq 0 .
$$


We are now going to derive an upper bound for $H=\max (U, W)$, where $W=\max _{j, q}\left|w_{j q}\right|$. First suppose that $c_{10} s \log P \cdot U>H$ with a sufficiently large $c_{10}$. We may assume that $U \geqq c_{11} s \log P$ with a sufficiently large $c_{11}$, for other wise (21) immediately follows. We see from (19) that

$$
\infty>\operatorname{ord}_{\mathfrak{P}_{1}} \Lambda \geqq U-c_{12} s \log P \geqq c_{13} U \geqq \frac{c_{14}}{s \log P} H .
$$

Further, by (19) we have

$$
\Lambda=-\frac{\lambda_{2} \chi_{2} \varrho_{2}}{\lambda_{3} \chi_{3}} \eta_{1}^{w_{12}} \ldots \eta_{r}^{w_{r 2}} \mu_{1}^{u_{12}^{\prime}-u_{13}^{\prime}} \ldots \mu_{t}^{u_{t 2}^{\prime}-u_{t 3}^{\prime}}-1 .
$$

Applying now Theorem 4 of van der Poorten [15] to $A$, we obtain by (16)

$$
H<c_{15}\left(c_{16} s\right)^{12(r+s f)+28} P^{g}(\log P)^{s f+4} .
$$

Suppose now that $c_{10} s \log P \cdot U \leqq H$. Assume, for convenience, that $W=\left|w_{11}\right|$. From (18) we conclude

$$
w_{11} \log \left|\eta_{1}^{(i)}\right|+\ldots+w_{r 1} \log \left|\eta_{r}^{(i)}\right|=\log \left|\delta_{1}^{(i)}\right|-\log \left|\chi_{1}^{(i)}\right|-\sum_{k} u_{k 1}^{\prime} \log \left|\mu_{k}^{(i)}\right|
$$

for each conjugate with $i=1, \ldots, r$. So for some $h$ we must have

Thus, by (16) we obtain

$$
W \leqq c_{17}\left(|\log | \delta_{1}^{(h)}||+|\log | \chi_{1}^{(h)}||+\sum_{k} u_{k 1}^{\prime}|\log | \mu_{k}^{(h)}||\right) .
$$

$$
|\log | \delta_{1}^{(h)}|| \geqq c_{18} W-c_{19} s \log P-c_{20} U s \log P \geqq c_{21} H,
$$

provided that $c_{10}$ is sufficiently large. Further, by (16) and (18) we have

$$
\log \left|N_{G / Q}\left(\delta_{1}\right)\right| \leqq \log \left|N_{G / Q}\left(\chi_{1}\right)\right|+U \cdot \sum_{k} \log \left|N_{G / Q}\left(\mu_{k}\right)\right| \leqq c_{22} U s \log P .
$$

Hence we get for some $m$

$$
\log \left|\delta_{1}^{(m)}\right| \leqq-c_{23} H .
$$

Formulae (16) and (18) imply

$$
\log \left|\frac{\lambda_{1}^{(m)}}{\lambda_{3}^{(m)} \delta_{3}^{(m)}}\right| \leqq c_{24}+(g-1) \log \mid \overline{\delta_{3} \mid} \leqq c_{25} U_{S} \log P<\frac{c_{23}}{2} H .
$$

We now omit the superscript $(m)$. It then follows from (22) and (23) that

$$
\log |\Lambda|<-\frac{c_{23}}{2} H \text {. }
$$

Write $\eta_{0}=-1$. By taking the principal values of the logarithms we obtain from (19) and (18)

$$
\begin{gathered}
0<\left|\log \left(-\frac{\lambda_{2} \delta_{2}}{\lambda_{3} \delta_{3}}\right)\right| \\
=\left|\sum_{j=0}^{r} w_{j 2} \log \eta_{j}+\sum_{k=1}^{t}\left(u_{k 2}^{\prime}-u_{k 3}^{\prime}\right) \log \mu_{k}-\log \left(-\frac{\lambda_{3} \chi_{3}}{\lambda_{2} \chi_{2} \varrho_{2}}\right)\right|<e^{-\delta^{*}(r+t+1) H},
\end{gathered}
$$


where $\delta^{*}=\left(c_{26}(r+t+1)\right)^{-1}$ and $w_{02}$ is a rational integer satisfying

$$
\left|w_{02}\right| \leqq(r+t+1) H .
$$

We can now apply Theorem 3 of van der Poorten and Loxton [16] to (24) and obtain

$$
H<c_{27}\left(c_{28} s\right)^{10(r+s f)+33}(\log P)^{s f+3} .
$$

So (21) and (25) imply

$$
H<c_{29}\left(c_{30} s\right)^{12(r+s f)+31} P^{g}(\log P)^{s f+4}
$$

and, by (16), (18) and (26), we have

$$
\begin{gathered}
\overline{\left|\delta_{q}\right|}<\exp \left\{c_{31} s \log P+c_{32} H+c_{33} H s \log P\right\}< \\
<\exp \left\{c_{34}\left(c_{30} s\right)^{12(r+s f)+32} P^{g}(\log P)^{s f+5}\right\}=T_{1}, \quad q=1,2,3 .
\end{gathered}
$$

Consider now any $\beta_{j}$ with $3 \leqq j \leqq n$. By the assumption made on $L_{1}^{\prime}, \ldots, L_{n}^{\prime}$ there is a sequence $\beta_{2}=\beta_{i_{1}}, \ldots, \beta_{i_{v}}=\beta_{j}$ such that for each $u$ with $1 \leqq u \leqq v-1$

$$
\lambda_{i_{u}} \beta_{i_{u}}+\lambda_{i_{u+1}} \beta_{i_{u+1}}+\lambda_{i_{u, u+1}} \beta_{i_{u, u+1}}=0
$$

holds with some non-zero $\lambda_{i_{u}}, \lambda_{i_{u+1}}, \lambda_{i_{u, u+1}} \in \boldsymbol{Z}_{G}$ satisfying $\max \left(\left|\overline{\lambda_{i_{u}}}\right|,\left|\overline{\lambda_{i_{u+1}}}\right|,\left|\overline{\lambda_{i_{u}, u+1}}\right|\right) \leqq$ $c_{35}$. Further, we may assume $v \leqq n$. We can see in the same way as above that

$$
\beta_{1}=\sigma \delta_{1}, \quad \beta_{2}=\sigma \delta_{2}
$$

and

$$
\beta_{i_{u}}=\sigma_{u} \delta_{u, i_{u}}, \quad \beta_{i_{u+1}}=\sigma_{u} \delta_{u, i_{u+1}}
$$

for $u=1, \ldots, v-1$, where $\delta_{u, i_{u}}, \delta_{u, i_{u+1}} \in \boldsymbol{Z}_{G}$ with

$$
\max _{1 \leqq u \leqq v-1}\left(\left|\overline{\delta_{u, i_{u}}}\right|,\left|\overline{\delta_{u, i_{u+1}}}\right|\right)<T_{1}
$$

and $\sigma_{u}=\vartheta_{u} \mu_{1}^{a_{1 u}} \ldots \mu_{t}^{a_{t u}}$ with units $\vartheta_{u} \in G$ and non-negative rational integers $a_{1 u}, \ldots, a_{t u}$. It follows from (28) and (29) that

$$
\beta_{j}=\beta_{i_{v}}=\sigma \varphi_{j} / \psi_{j}
$$

with

$$
\varphi_{j}=\delta_{2} \prod_{u=1}^{v-1} \delta_{u, i_{u+1}} \quad \text { and } \quad \psi_{j}=\prod_{u=1}^{v-1} \delta_{u, i_{u}}
$$

Write $\psi_{1}=\psi_{2}=1$ and $\varphi_{j}=\delta_{j}$ for $j=1,2$. It is clear that

$$
\max \left(\left|\overline{\varphi_{j}}\right|,\left|\overline{\psi_{j}}\right|\right)<T_{1}^{n}, \quad j=1, \ldots, n .
$$

We recall that $\sigma=\varepsilon_{3} \mu_{1}^{a_{1}} \ldots \mu_{t}^{a_{\mathrm{t}}}$. Denote by $\mu_{k}^{b_{k}}$ the highest power of $\mu_{k}$ with $b_{k} \leqq a_{k}$ that divides at least one of the $\psi_{1}, \ldots, \psi_{n}$. By taking norms we see that

$$
b_{k} \leqq c_{36} \log T_{1}, \quad k=1, \ldots, t .
$$


Putting

and

$$
b_{k}^{*}=\min \left(a_{k}, b_{k}+1\right), \quad d_{k}=a_{k}-b_{k}^{*}, \quad k=1, \ldots, t,
$$

we get

$$
\tau_{j}=\mu_{1}^{b_{1}^{*}} \ldots \mu_{t}^{b_{t}^{*}} \varphi_{j} / \psi_{j},
$$

$$
\beta_{j}=\vartheta \mu_{1}^{d} \ldots \mu_{t}^{d} \tau_{j}, \quad j=1, \ldots, n,
$$

where $\vartheta=\varepsilon_{3}$ is a unit and $\tau_{j}$ are algebraic integers in $G$ satisfying

$$
\left|\overline{\tau_{j}}\right|<\exp \left\{c_{37} s \log P \log T_{1}\right\}=T_{2} .
$$

Further, by (13) we have

$$
\mathfrak{p}_{1}^{v_{1}} \ldots \mathfrak{p}_{s}^{v_{s}}=\left(\beta_{1} \ldots \beta_{n}\right)=\left(\left(\vartheta \mu_{1}^{d_{1}} \ldots \mu_{t}^{d^{t}}\right)^{n} \tau_{1} \ldots \tau_{n}\right) .
$$

Let $k, 1 \leqq k \leqq s$, be an arbitrary but fixed subscript, and let $\mathfrak{P}$ denote an arbitrary prime ideal in $G$ lying above $\mathfrak{p}_{k}$. If $\mathfrak{P}^{\boldsymbol{e}_{k}} \| \mathfrak{p}_{k}, e_{k}$ does not depend on the choice of $\mathfrak{P}$. Moreover, $\mathfrak{P}$ divides only one of the $\mu_{1}, \ldots, \mu_{t}$. We shall now follow an argument used in the proof of Theorem 1 of [5] (cf. the deduction (36) $\Rightarrow(41)$ of [5]). Let $y_{k}$ be the greatest rational integer for which

$$
\min \left(v_{k} e_{k}-\operatorname{ord}_{\mathfrak{P}}\left(\prod_{j=1}^{n} \tau_{j}\right), v_{k} e_{k}\right) \geqq n h_{L} y_{k} e_{k}
$$

holds for each $\mathfrak{P}$ with $\mathfrak{P} \mid \mathfrak{p}_{k}$, where $h_{L}$ denotes the class number of $L$. From (35) it follows that $y_{k} \geqq 0$. By the definition of the $y_{k}$ there is a $\mathfrak{P}$, lying above $\mathfrak{p}_{k}$, such that

$$
n h_{L}\left(y_{k}+1\right) e_{k}>\min \left(v_{k} e_{k}-\operatorname{ord}_{\mathfrak{P}}\left(\prod_{j=1}^{n} \tau_{j}\right), v_{k} e_{k}\right) .
$$

Since (34) implies

$$
\operatorname{ord}_{\mathfrak{P}}\left(\prod_{j=1}^{n} \tau_{j}\right) \leqq c_{38} \log T_{2}
$$

we get from (36) and (37)

$$
0 \leqq v_{k} e_{k}-n h_{L} y_{k} e_{k} \leqq c_{39} \log T_{2} .
$$

If now $\mathfrak{P}$ is an arbitrary prime ideal in $G$ lying above $\mathfrak{p}_{k}$ and $\mathfrak{P} \mid\left(\mu_{p}\right)$, then (35), (36) and (38) give

$$
0 \leqq d_{p} \operatorname{ord}_{\mathfrak{P}} \mu_{p}-h_{L} y_{k} e_{k} \leqq c_{40} \log T_{2} .
$$

Let now $\mathfrak{p}_{1}^{h_{L}} y_{1} \ldots \mathfrak{p}_{s}^{h_{L}} y_{s}=(\varkappa)$, where $x \in Z_{L}$, and choose $\xi$ in such a way that

$$
\mu_{1}^{d_{1}} \ldots \mu_{t}^{d_{t}}=\varkappa \xi \text {. }
$$

In view of (39) $\xi$ is an algebraic integer in $G$ and

$$
\left|N_{G / Q}(\xi)\right| \leqq \exp \left\{c_{41} s \log P \log T_{2}\right\} .
$$


It follows from (33) and (40) that

$$
\omega=\vartheta^{n} \xi^{n} \tau_{1} \ldots \tau_{n} \in Z_{L} .
$$

Further, Lemma 3 of [6] together with (34) and (41) imply that there is a unit $\theta_{1} \in L$ and an $\omega^{\prime} \in Z_{L}$ such that

and

$$
\omega=\theta_{1}^{n} \omega^{\prime}
$$

$$
\left|\overline{\omega^{\prime}}\right|<\exp \left\{c_{42} s \log P \log T_{2}\right\}
$$

Thus by (34) and (42) we have

$$
\left|\overline{\theta_{1}^{-1} \vartheta \xi}\right|<\exp \left\{c_{43} s \log P \log T_{2}\right\} .
$$

Finally, writing $\xi_{j}=\theta_{1}^{-1} \vartheta \xi_{j}$ we get

$$
\beta_{j}=\theta_{1} x \xi_{j}, \quad j=1, \ldots, n,
$$

and, by (34) and (43),

$$
\left|\overline{\xi_{j}}\right|<\exp \left\{c_{44} s \log P \log T_{2}\right\}=T_{3} .
$$

By hypothesis there is no $0 \neq \mathbf{x} \in L^{m}$ for which $L_{j}^{\prime}(\mathbf{x})=0, j=1, \ldots, n$. Consequently, the only solution in $L$ of the system of equations

$$
L_{j}^{\prime}(\mathbf{x})=\beta_{j}, \quad j=1, \ldots, n,
$$

is the $\mathbf{x}=\left(x_{1}, \ldots, x_{m}\right)$ considered above. Since $f(\mathbf{x}) / a_{0}^{n}$ is a product of irreducible norm forms over $L,(46)$ contains all conjugates of each equation over $L$. Following now an argument of the proof of Lemma 2 of [7], we can easily see that (46) has no other solutions in the complex field. So $m \leqq n f$, and by Cramer's rule we have

$$
x_{i}=\theta_{1} \varkappa v_{i} / v, \quad i=1, \ldots, m,
$$

where $v, v_{i} \in \boldsymbol{Z}_{G}, v_{1}, \ldots, v_{m}$ are not all zero,

$$
\overline{|v|} \leqq c_{45}
$$

and, by (45),

$$
\overline{\left|v_{i}\right|} \leqq c_{46} T_{3}, \quad i=1, \ldots, m .
$$

In view of (47) we obtain in $Z_{G}$

$$
\left|N_{G / Q}(\varkappa)\right| N\left(\left(v_{1}, \ldots, v_{m}\right)\right)=\left|N_{G / Q}(v)\right| N\left(\left(x_{1}, \ldots, x_{m}\right)\right) .
$$

Hence, by (48),

$$
\left|N_{L / Q}(\varkappa)\right| \leqq\left|N_{G / Q}(v)\right|^{1 / f} d \leqq c_{47} .
$$

Thus we can write $\theta_{1} \varkappa=\theta_{2}^{-1} \varkappa^{\prime}$ with a unit $\theta_{2} \in L$ and an algebraic integer $\varkappa^{\prime} \in L$ satisfying

$$
\left|\overline{\varkappa^{\prime}}\right| \leqq c_{48} .
$$


It follows now from (47) that

and this implies

$$
x_{i}^{\prime}=\theta_{2} x_{i}=\varkappa^{\prime} v_{i} / v, \quad i=1, \ldots, m,
$$

$$
x_{i}^{\prime f}=N_{G / L}\left(x_{i}^{\prime}\right)=N_{G / L}\left(\varkappa^{\prime} v_{i}\right) / N_{G / L}(v), \quad i=1, \ldots, m .
$$

By the inequality (24) of [7] we have

$$
\left|\overline{x_{i}^{\prime}}\right|^{f} \leqq \overline{N_{G / L}\left(\varkappa^{\prime} v_{i}\right)}\left|\overline{N_{G / L}(v)}\right|^{l-1} \leqq\left.\overline{\varkappa^{\prime} v_{i}}\right|^{f} \mid \overline{v \mid}^{(l-1) f},
$$

whence, by (48), (49), (51), (45), (34) and (27) we obtain

$$
\max _{1 \leqq i \leqq m}\left|\overline{x_{i}^{\prime}}\right|<c_{49} T_{3} \leqq \exp \left\{c_{50}\left(c_{51} s\right)^{12(r+s f)+34} P^{g}(\log P)^{s f+7}\right\} .
$$

From (52) we deduce

(53) $\log \log |\overline{\mathbf{x}}|<\log c_{50}+(12(r+s f)+34) \log \left(c_{51} s\right)+g \log P+(s f+7) \log \log P$.

If $X_{4}$ is sufficiently large, then $P$ is also sufficiently large and $s>(\log P)^{3 f /(3 f+1)}$ implies

$$
\begin{gathered}
\log c_{50}+(12(r+s f)+34) \log c_{51}+(12 r+34) \log (s+1)+(s f+7) \log \log P \\
\leqq\left(f+\frac{1}{2}\right) s \log (s+1) .
\end{gathered}
$$

On the other hand, for $s \leqq(\log P)^{3 f /(3 f+1)}$ we have

$$
\log c_{50}+(12(r+s f)+34) \log c_{51}+(12 r+34) \log (s+1)+(s f+7) \log \log P \leqq \log P .
$$

Hence (53) gives

$$
\log \log \overline{|\mathbf{x}|}<\left(13 f+\frac{1}{2}\right) s \log (s+1)+(g+1) \log P,
$$

whence (4) follows.

By prime number theory we can choose $X_{4}$ such that even $\pi(P) \leqq(1+\delta) P / \log P$ holds with $\delta=1 /(2(26 f+1))$. Then $s \leqq l \pi(P) \leqq(1+\delta) l P / \log P$ and thus

$$
\left(13 f+\frac{1}{2}\right) s \log (s+1)+(g+1) \log P \leqq(13 f+1) l P .
$$

Finally, in consequence of (54), (55) and $\mathscr{P}=P^{\alpha}$ we obtain (5).

In order to prove $\left(4^{\prime}\right)$ and $\left(5^{\prime}\right)$ it suffices to observe that (53) and (3) imply

$\log \log N<\log \left(l c_{50}\right)+(12(r+s f)+34) \log \left(c_{51} s\right)+g \log P+(s f+7) \log \log P$.

If $N$ is sufficiently large, we get $\left(4^{\prime}\right)$ and $\left(5^{\prime}\right)$ in the same way as we deduced (4) and (5) from (53). 


\section{Proofs of the Corollaries}

Proof of Corollary 1. Let $\varepsilon$ be a unit in $L$ such that $\overline{\mid \mathbf{x}} \mid=\max \left(\left|\overline{\varepsilon x_{1}}\right|, \ldots,\left|\overline{\varepsilon x_{m}}\right|\right)$. Then

$$
N=\left|N_{L / Q}(F)\right|=\left|N_{L / Q}(F(\varepsilon \mathbf{x}))\right| \leqq c_{52}|\overline{\mathbf{x}}|^{n l} .
$$

Therefore, for sufficiently large $N$, (4) implies (7), but only with $\log \log N-\log (2 \ln )$ in place of $\log \log N$. Following the argument applied at the end of the above proof, we obtain (7) and (8) from (53) and (56).

Proof of Corollary 2. Suppose

$$
\omega(F(\mathbf{x})) \leqq c_{4} \frac{\log \log \overline{|\mathbf{x}|}}{\log \log \log \overline{|\mathbf{x}|}}
$$

for some $\mathbf{x} \in \boldsymbol{Z}_{L}^{m}$ with $\overline{|\mathbf{x}|} \geqq X_{5}$ and $N\left(\left(x_{1}, \ldots, x_{m}\right)\right) \leqq d$. Then by our theorem we have

$$
\begin{aligned}
\log \log \overline{\mid \mathbf{x}} \mid & <(13 f+1) \omega(F(\mathbf{x})) \log (\omega(F(\mathbf{x}))+1)+(g+1) \log \mathscr{P}(F(\mathbf{x})) \\
& \leqq(13 f+1) c_{4} \log \log \overline{|\mathbf{x}|}+A(g+1) \log \log \overline{|\mathbf{x}|},
\end{aligned}
$$

provided that $X_{5}$ is sufficiently large. Since $(13 f+1) c_{4}+A(g+1)=1$, we have arrived at a contradiction and thus (9) is proved.

Proof of Corollary 3. By assumption there are at least three pairwise nonproportional linear factors in the factorization

$$
f(x, y)=\prod_{i=1}^{n}\left(\alpha_{i 1} x_{1}+\alpha_{i 2} y\right) .
$$

Consequently, the linear factors $\alpha_{i 1} x+\alpha_{i 2} y, i=1, \ldots, n$, form a $\Delta$-connected system and the system of equations

$$
\alpha_{i 1} x+\alpha_{i 2} y=0, \quad i=1, \ldots, n,
$$

has no non-trivial solution $x, y$ in $L$. So the assertion of Corollary 3 follows at once from our theorem.

Proof of Corollary 4. $F(\mathbf{x})$ can be written in the form

$$
F(\mathbf{x})=a_{0} \prod_{i=1}^{n}\left(x_{1}+\alpha_{2}^{(i)} x_{2}+\ldots+\alpha_{m}^{(i)} x_{m}\right),
$$

where $\alpha_{j}^{(1)}, \ldots, \alpha_{j}^{(n)}$ denote the conjugates of $\alpha_{j}$ over $L$. As we showed in [7] (see also [9]), the conjugates $x_{1}+\alpha_{2}^{(i)} x_{2}+\ldots+\alpha_{m}^{(i)} x_{m}$ of $x_{1}+\alpha_{2} x_{2}+\ldots+\alpha_{m} x_{m}$ over $L$ form a $\Delta$-connected system. Further, by virtue of the assumption $\left[L\left(\alpha_{1}\right): L\right] \ldots$ $\left[L\left(\alpha_{m}\right): L\right]=n$, the only solution of the system of equations

$$
x_{1}+\alpha_{2}^{(i)} x_{2}+\ldots+\alpha_{m}^{(i)} x_{m}=0, \quad i=1, \ldots, n,
$$


in $L$ is $x_{1}=\ldots=x_{m}=0$. So our theorem implies the required assertion.

Proof of Corollary 5. Let $L(\mathbf{x})=\alpha_{1} x_{1}+\ldots+\alpha_{m} x_{m}$ and let $L^{(1)}(\mathbf{x}), \ldots, L^{(n)}(\mathbf{x})$ be the conjugates of $L(\mathbf{x})$ over $L$. Put

$$
l_{i j}(\mathbf{x})=L^{(i)}(\mathbf{x})-L^{(j)}(\mathbf{x}) .
$$

In proving Theorem 4 in [7] we showed that

$$
F(\mathbf{x})=\operatorname{Discr}_{K / L}\left(\alpha_{1} x_{1}+\ldots+\alpha_{m} x_{m}\right)=(-1)^{n(n-1) / 2} \prod_{\substack{i, j=1 \\ i \neq j}}^{n} l_{i j}(\mathbf{x})
$$

satisfies all conditions made in our theorem. Thus (4) and (5) clearly follow.

Proof of Corollary 6. If $X_{8}$ is sufficiently large and $\overline{\mid \mathbf{x}} \mid \geqq X_{8}$, by Corollary 5 and (11) we have $\mathscr{P}(D(\mathbf{x}))=\mathscr{P}(I(\mathbf{x}))$, where $D(\mathbf{x})=\operatorname{Discr}_{K / L}\left(\alpha_{1} x_{1}+\ldots+\alpha_{n-1} x_{n-1}\right)$. Thus Corollary 5 proves the assertion of Corollary 6 .

\section{References}

[1] BAKer, A.: Contributions to the theory of Diophantine equations. I and II. - Philos. Trans. Roy. Soc. London Ser. A 263, 1967/68, 173-208.

[2] Berwick, W. E. H.: Integral bases. - Reprinted by Stechert - Hafner Service Agency, New York-London, 1964.

[3] Borevich, Z. I., and I. R. Shafarevich: Number theory. - Academic Press, New York-London, 1967.

[4] CoAtes, J.: An effective $p$-adic analogue of a theorem of Thue. II. The greatest prime factor of a binary form. - Acta Arith. 16, 1969/70, 399-412.

[5] GYÖRY, K.: On polynomials with integer coefficients and given discriminant, V. p-adic generalizations. - Acta Math. Acad. Sci. Hungar. 32, 1978, 175-190.

[6] GYőRY, K.: On the solutions of linear diophantine equations in algebraic integers of bounded norm. - Ann. Univ. Sci. Budapest. Eötvös Sect. Math. (to appear).

[7] GYőRY, K., and Z. Z. PAPP: Effective estimates for the integer solutions of norm form and discriminant form equations. - Publ. Math. Debrecen 25, 1978, 311-325.

[8] Győry, K., and Z. Z. PAPP: On discriminant form and index form equations. - Studia Sci. Math. Hungar. (to appear).

[9] GyőRY, K., and Z. Z. PAPP: Norm form equations and explicit lower bounds for linear forms with algebraic coefficients. - To appear.

[10] GYóRY, K.: Effective upper bounds for the solutions of norm form equations in the $p$-adic case. - Preprint, 1978.

[11] Koтоv, S. V.: The Thue-Mahler equation in relative fields. - Collection of articles in memory of Juriǐ Vladimirovič Linnik, Acta Arith. 27, 1975, 293-315 (Russian).

[12] MAHLER, K.: Zur Approximation algebraischer Zahlen, I. Über den grössten Primteiler binärer Formen. - Math. Ann. 107, 1933, 691-730.

[13] Narkiewicz, W.: Elementary and analytic theory of algebraic numbers. - PWN-Polish Scientific Publishers, Warszawa, 1974.

[14] Parry, C. J.: The p-adic generalization of the Thue-Siegel theorem. - Acta Math. 83, 1950, $1-100$. 
[15] VAn Der Poorten, A. J.: Linear forms in logarithms in the p-adic case. - Transcendence Theory: Advances and Applications, edited by A. Baker and D. W. Masser, Academic Press, London-New York-San Francisco, 1977, 29-57.

[16] VAn DeR Poorten, A. J., and J. H. Loxton: Multiplicative relations in number fields. - Bull. Austral. Math. Soc. 16, 1977, 83-98 and 17, 1977, 151-156.

[17] Schlickewei, H. P.: On norm form equations. - J. Number Theory 9, 1977, 370-380.

[18] Schlickewei, H. P.: On linear forms with algebraic coefficients and Diophantine equations. J. Number Theory 9, 1977, 381-392.

[19] Shorey, T. N., and R. TiJdeman: On the greatest prime factors of polynomials at integer points. - Compositio Math. 33, 1976, 187-195.

[20] Shorey, T. N., A. J. van der Poorten, R. Tijdeman, and A. Schinzel: Applications of the Gel'fond-Baker method to diophantine equations. - Transcendence Theory: Advances and Applications, edited by A. Baker and D. W. Masser, Academic Press, LondonNew York-San Francisco, 1977, 59-77.

[21] SprindžUK, V. G.: The greatest prime divisor of a binary form. - Dokl. Akad. Nauk BSSR 15, 1971, 389-391 (Russian).

[22] SPRindžUK, V. G.: The structure of numbers representable by binary forms. - Dokl. Akad. Nauk BSSR 17, 1973, 685-688, 775 (Russian).

[23] SPRINDžUK, V. G.: An effective analysis of the Thue and Thue-Mahler equations. - Current problems of analytic number theory, Proc. Summer School Analytic Number Theory, Minsk, 1972, pp. 199-222, 272, Izdat. "Nauka i Tehnika", Minsk, 1974 (Russian).

[24] TrelinA, L. A.: On the greatest prime factor of an index form. - Dokl. Akad. Nauk BSSR 21, 1977, 975-976 (Russian).

Added in proof. The results of this paper were presented with detailed proofs in my course given at the University of Paris VI, March-June 1979.

\author{
Kossuth Lajos University \\ Mathematical Institute \\ H-4010 Debrecen 10 \\ Hungary
}

Received 6 November 1978 